\title{
Regulation Method for Torque-Angle Characteristics of Rotary Electric-Mechanical Converter Based on Hybrid Air Gap
}

\author{
Bin Meng ${ }^{*}(\mathbb{D}$, Yongjiang Lai and Xinguo Qiu
}

\begin{abstract}
The torque-angle characteristics of electric-mechanical converters are important determinants of the quality of electrohydraulic proportional control systems. It is far more difficult for a rotary electric-mechanical converter (REMC) to obtain flat torque-angle characteristics than traditional proportional solenoid, greatly influencing the promotion and application of rotary valves for electrohydraulic proportional control systems. A simple and feasible regulation method for the torque-angle characteristics of REMCs based on a hybrid air gap is proposed. The regulation is performed by paralleling an additional axial air gap with the original radial air gap to obtain a flat torque-angle characteristic and increase output torque. For comparison, prototypes of REMCs based on hybrid and radial air gaps were manufactured, and a special test rig was built. The torque-angle characteristics under different excitation currents and step responses were studied by magnetic circuit analysis, finite element simulation, and experimental research. The experimental results were consistent with the theoretical analysis. It was shown that REMCs based on a hybrid air gap can obtain a flat torque-angle characteristic with further optimizing of key structural parameters and also increase output torque. This regulation method provides a new approach for the design of proportional rotary electromechanical converters.
\end{abstract}

Keywords: Rotary valve, Rotary electric-mechanical converter, Torque-angle characteristic, Hybrid air gap, Axial air gap, Radial air gap

\section{Introduction}

Since its advent, electrohydraulic proportional control technology has occupied a top position in the electromechanical industry because it has the advantages of a high power to weight ratio, large output force, and excellent static and dynamic characteristics, which are regarded as key to competitiveness in modern industry [1-7]. As a crucial control component, the electrohydraulic proportional valve has a decisive influence on a system's overall performance and, therefore, is always a focus of research on fluid power transmission and control [8-11]. A rotary valve is one such valve, and it utilizes rotary motion to

\footnotetext{
*Correspondence: bin_meng@zjut.edu.cn

Key Laboratory of Special Purpose Equipment and Advanced Processing

Technology, Ministry of Education, Zhejiang University of Technology,
} Hangzhou, China change the relative position of the valve core and sleeve to realize opening, closing, and reversing of the fluid flow path [12-14]. Compared with spool and poppet valves, a rotary valve features high reliability, simple structure, high working frequency, and excellent antioil pollution ability, and it is expected to be widely used in high-speed excitation and commutation hydraulic systems [15-18]. Moreover, a single-stage rotary valve's flow rate can be much greater than that of a two-stage spool valve, as it has multiple throttling grooves [19]. However, in the modern hydraulic industry, the application of rotary valves is far less extensive than that of spool valves. The main reason is that, it is far more difficult for the rotary electric-mechanical converter (REMC) used to drive the rotary valve to obtain flat torque-angle characteristics than it is for the linear proportional solenoid of the spool valve. For the latter, by designing a magnetic isolation 
ring structure, the solenoid's magnetic circuit can be easily divided into axial and radial subcomponents, where, after synthesis, the flat force-displacement characteristic can be obtained to realize proportional control [20-22]. Although the welding process for the magnetic isolation ring is complicated and time-consuming, it is not a serious issue for large-scale automation production. For an REMC, there is no such general method to obtain proportional control characteristics.

Flat torque-angle characteristics of REMC are one of the important determinants of the quality of an electrohydraulic proportional control system [23, 24]. Much research has been done on optimization for the magnetic circuit topology of REMC. According to the working principles, an REMC can be divided into voice coil motor, torque motor, rotary electromagnet, etc. Wu et al. proposed a rotary voice coil motor for a direct-driven valve based on a Halbach array, whose torque-current characteristics have very good linearity. However, its output torque is only about $0.05 \mathrm{~N} \cdot \mathrm{m}$ owing to magnetic topology [25]. Buscher et al. [26] proposed a dual-E stator-type torque motor, which can provide larger coil placement space than a traditional torque motor. Also, the permanent magnet (PM) is closer to the working air gap, so that the bias flux path is much shorter, and the loss of bias flux leakage is reduced. For torque motors used in nozzle flapper valves and jet-pipe servo valves, proportional characteristics can also be obtained through reasonable design of the spring components [27]. However, it is difficult to have a larger working stroke, because its magnetic circuit is totally based on the axial air gap. The rotary electromagnet proposed by Montagu broadens working angle range by using the radial air gap to replace the axial air gap, yet the torque-angle characteristics show a monotonous downward trend [28]. To obtain a flat torque-angle curve, Hitachi proposed a special design for PM shape on the rotor of the torque motor, where the pole surface is cut along the radial direction and filled with nonmagnetic material to compensate for torque ripple [29]. The Denso company also proposed a PM-type torque motor, where two magnetic poles composed of the discrete PMs are asymmetrically arranged on the outer side of a rotating shaft by using the half-pole angle difference between them to compensate for the torque ripple caused by the polygonal magnetic pole [30]. Zhang et al. [31] proposed an electric excitation rotary electromagnet whose stator yoke shape and rotor pole surface were specially designed to control the magnetic flux saturation at the stator yoke top to obtain a flat torque-angle characteristic. Cui et al. [32] proposed a rotary proportional electromagnet based on a radial working air gap. It is based on a differential magnetic circuit, and its structure is too complex for industrial application and mass production. There are also some commercially available REMCs. The GDA and GDR series rotary proportional electromagnets manufactured by the SCHULTZ Company have very large working angles and can be equipped with angular displacement sensors to improve control accuracy, but the details of magnetic circuit are not disclosed.

In this study, a simple and feasible regulation method for torque-angle characteristics of REMCs based on a hybrid air gap was developed by paralleling an additional axial air gap to obtain flat torque-angle characteristics. To validate the practicability of this method, prototypes of the REMC based on hybrid and radial air gaps were manufactured, and their torque-angle characteristics and step responses were analyzed through magnetic circuit analysis, finite-element method (FEM) simulation, and experimental approaches.

\section{Working Principle and Structure}

There are two kinds of working air gap commonly used for REMC design: radial and axial air gap. However, the torque generation mechanisms are quite different. An REMC with a radial air gap (REMC-R) has a larger working angle, but with the alignment of the stator yoke and rotor tooth, the output torque decreases gradually, i.e., the slope of the torque-angle curve is negative. The working angle of the REMC with an axial air gap (REMC-A) is quite limited, but the output torque greatly increases when the armature gets close to the stator yoke, i.e., the slope of the torque-angle characteristic curve is sharply positive.

Therefore, if the features of both the radial and axial air gap can be utilized, i.e., if an REMC with a hybrid air gap (REMC-H) can be formed by paralleling an additional axial air gap with a radial air gap, as shown in Figure 1, where the torque generated by the radial and axial air gaps can be mutually modulated and compensated for, then a flat torque, angle curve can be expected. In addition, the paralleled axial air gap can increase the output torque, because it provides an extra path for magnetic flux.

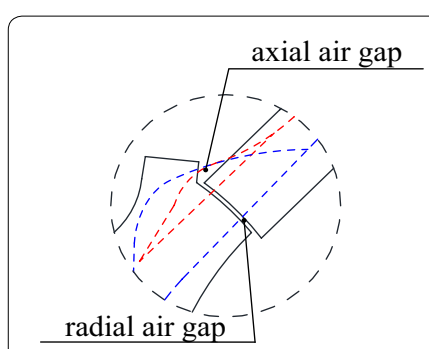

(a) REMC-H

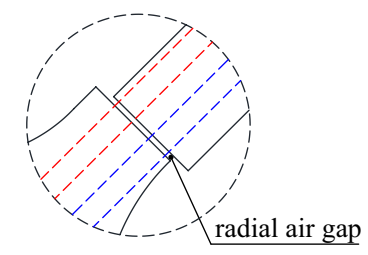

(b) REMC-R
Figure 1 Schematic of two different air gaps 


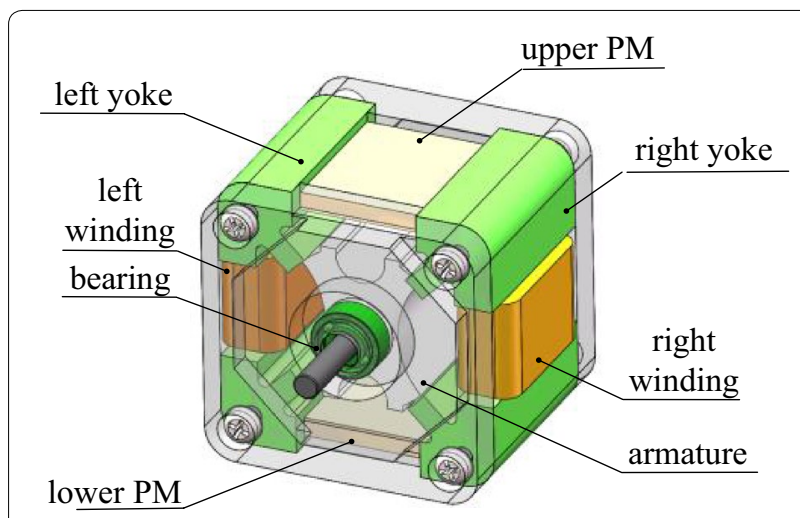

Figure 2 Structure schematic of REMC-H

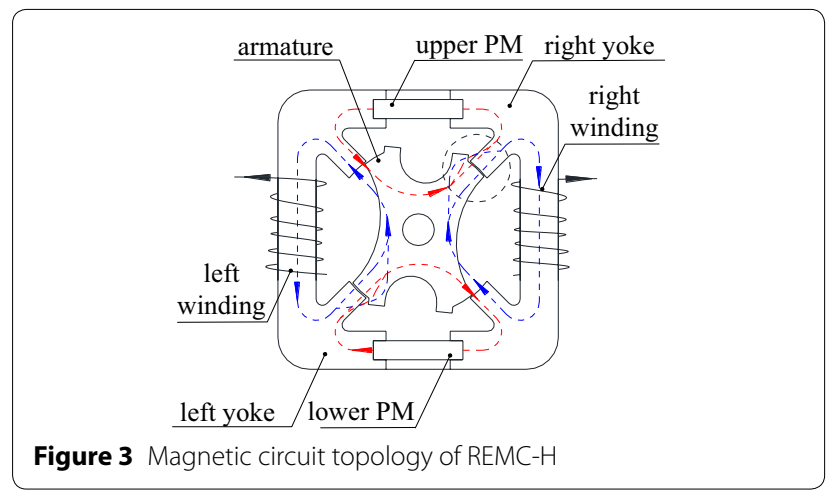

Figures 2 and 3 show a structure schematic and the magnetic circuit of the proposed RMEC-H. It consists of stator and armature components. The former consists of a left and right stator yoke where symmetrical notches in the middle of the stator yoke are machined to provide space for coil winding to produce a control magnetic field. Each stator yoke has upper and lower poles, and four poles are distributed on the diagonal line $90^{\circ}$ apart. The upper and lower PMs are embedded in the stator yoke slot to provide a bias magnetic field. The armature component is composed of armature and shaft. The armature has four large teeth uniformly distributed along the radial direction. The end face of each tooth is composed of two parts. The first part is an arc annular surface, which forms a radial air gap with the stator yoke. The second part is a rectangular block, which distributes at the end of the arc surface with large teeth and thus forms an axial air gap with the side of the stator yoke. When the REMC-H is not electrified, the armature is in the equilibrium position with an external linear spring. When current flows through the winding, the control flux and the bias flux interact, and magnetic torque is generated, whose magnitude and direction can be controlled by the excitation current.

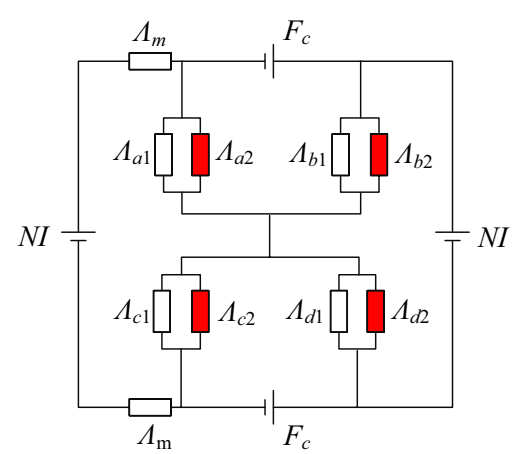

Figure 4 Equivalent magnetic circuit diagram of REMC-H
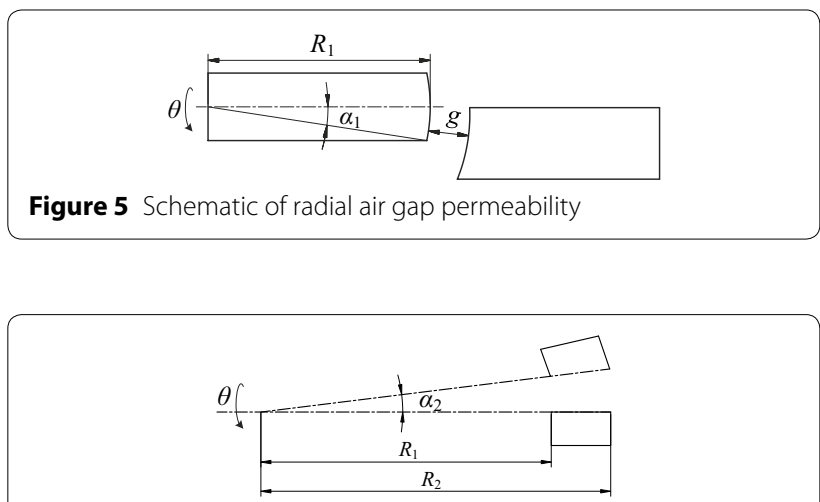

Figure 6 Schematic of axial air gap permeability

\section{Magnetic Circuit Analysis}

To obtain torque-angle equations for the REMC-H and analyze the influence of key structure and operation parameters on torque-angle characteristics, the equivalent magnetic circuit method was used for analysis. Assuming that the soft magnetic material works in the linear region and the permeability of stator and armature is infinite, it can be considered that the magnetic potential drop is mainly concentrated on the air gap.

The equivalent magnetic circuit diagram of the REMC$\mathrm{H}$ is shown in Figure 4. The permeability of each air gap is denoted as $\Lambda_{a}, \Lambda_{b}, \Lambda_{c}$, and $\Lambda_{d}$, each of which can be further decomposed into radial and axial permeability components. Figures 5 and 6 are schematics of radial air gap permeability and axial air gap permeability, respectively, where $g$ is the radial length of the air gap, $R_{1}$ is armature radius, $R_{2}$ is the distance between the armature center and its top, $\alpha_{1}$ is the initial position angle of the radial air gap, $\alpha_{2}$ is the initial position angle of the axial air gap, $\theta$ is the rotating angle of the armature, and $F_{c}$ is the magnetic potential of the PM.

When the armature rotates clockwise, $\Lambda_{a}$ equals radial permeability component $\Lambda_{a 1}$, and the axial permeability 
component $\Lambda_{a 2}$ is nearly zero. Also, $\Lambda_{b}$ equals the sum of $\Lambda_{b 1}$ and $\Lambda_{b 2}$. The analysis of $\Lambda_{c}$ and $\Lambda_{d}$ is similar. Thus,

$$
\begin{aligned}
& \Lambda_{a}=\Lambda_{a 1}=\Lambda_{c}, \\
& \Lambda_{b}=\Lambda_{b 1}+\Lambda_{b 2}=\Lambda_{d} .
\end{aligned}
$$

According to Figures 5 and 6, and neglecting the reluctance of the PM,

$$
\begin{aligned}
& \Lambda_{a 1}=\frac{\mu_{0} b\left(2 R_{1}+g\right)\left(a_{1}-\theta\right)}{2 g}, \\
& \Lambda_{b 1}=\frac{\mu_{0} b\left(2 R_{1}+g\right)\left(a_{1}+\theta\right)}{2 g}, \\
& \Lambda_{b 2}=\mu_{0} \frac{b}{a_{2}-\theta} \ln \frac{R_{2}}{R_{1}}=\frac{k_{2}}{\left(a_{2}-\theta\right)},
\end{aligned}
$$

where $\mu_{0}$ is air permeability and $b$ is the axial length of the air gap.

Assuming $\alpha=a_{1}=\alpha_{2}$, the magnetic potential drops $U_{a}$ and $U_{b}$ corresponding to $\Lambda_{a}$ and $\Lambda_{b}$ can be calculated as

$$
\left\{\begin{array}{l}
U_{a}=U_{m a}-U_{c a} \\
U_{b}=U_{m b}+U_{c b}
\end{array}\right.
$$

where

$$
\left\{\begin{array}{l}
U_{m a}=F_{c} \frac{\Lambda_{b}}{\Lambda_{a}+\Lambda_{b}}, \\
U_{m b}=F_{c} \frac{\Lambda_{a}}{\Lambda_{a}+\Lambda_{b}}, \\
U_{c a}=N I \frac{\Lambda_{b}}{\Lambda_{a}+\Lambda_{b}}, \\
U_{c b}=N I \frac{\Lambda_{a}}{\Lambda_{a}+\Lambda_{b}},
\end{array}\right.
$$

where $U_{m a}$ and $U_{m b}$ are magnetic potential drops corresponding to $\Lambda_{a}$ and $\Lambda_{b}$ with PM excitation alone, $U_{c a}$ and $U_{c b}$ are magnetic potential drops corresponding to $\Lambda_{a}$ and $\Lambda_{b}$ with current excitation alone, $N$ is the turn number of the coil, and $I$ is the excitation current.

With the assumption of a linear magnetic circuit, the total magnetic coenergy $w^{\prime}$ of the REMC-H can be expressed as

$$
w^{\prime}=\frac{1}{2} \sum_{i=1}^{4} U_{i}^{2} \Lambda_{i} .
$$

The magnetic output torque can be written as

$$
T=-\frac{\partial w^{\prime}}{\partial \theta} .
$$

Substituting Eqs. (1)-(8) into Eq. (9), the equation of torque-angle characteristics of REMC-H can be written as

$$
\begin{aligned}
T_{H}= & F_{c} N I \frac{2 k_{1} a^{2}+k_{1} k_{2}+2 k_{2}}{2 a^{2}}+\left[F_{c}^{2}+(N I)^{2}\right] \frac{k_{1} \theta}{a} \\
& -3 F_{c} N I \frac{k_{1}^{2} \theta^{2}}{a^{2}}+F_{c} N I \frac{k_{2}^{2}}{2 a^{2}(a-\theta)^{2}} .
\end{aligned}
$$

Similarly, by neglecting the axial air gap, the equation of the torque-angle characteristics of the REMC-R can be written as

$$
T_{R}=F_{c} N I k_{1}-\left[F_{c}^{2}+(N I)^{2}\right] \frac{k_{1} \theta}{a}-3 F_{c} N I \frac{k_{1}^{2} \theta^{2}}{a^{2}},
$$

where

$$
\begin{aligned}
& k_{1}=\frac{\mu_{0} b(2 R+g)}{2 g}, \\
& k_{2}=\mu_{0} b \ln \frac{R_{2}}{R_{1}},
\end{aligned}
$$

where $k_{1}$ is the coefficient of radial air gap permeability, $k_{2}$ is the coefficient of axial air gap permeability, and both represent the structure features of the axial and radial air gaps, respectively.

It can be seen from Eq. (11) that the equation of REMC$\mathrm{R}$ contains three terms, where the first term is independent of $\theta$, and, therefore, it remains unchanged when the current is constant. The second term is linear with $\theta$, and the third term is proportional to $\theta^{2}$, which indicates that the torque-angle curve is a monotonous curve decreasing with $\theta$. Because of $\theta^{2}$, the greater the $\theta$, the more the torque decreases. For REMC-H, Eq. (10) consists of four terms. The meanings of first three terms are similar to Eq. (11). The fourth term indicates that the compensation effect of the additional axial air gap on the torque is a quadratic parabolic relationship. With the increase of $\theta$, the term $(a-\theta)^{2}$ increases. When $\theta$ approaches $\alpha$, $(a-\theta)^{2}$ is infinitely close to zero, which indicates that the compensation torque increases with the increase of $\theta$, and the greater the $\theta$, the faster the torque increases. Therefore, with this fourth term, it is possible to change the monotonously decreasing torque-angle curve into a nearly flat one and also to increase the output torque. In addition, it can be seen from the first item of Eq. (11) that, for REMC-H, there exists an evident coupling relationship between $k_{1}$ and $k_{2}$. Thus, from the analytic equation, it is difficult to know which structural parameters need to be selected out to do the parametric design, and help from FEM simulation is needed. 


\section{FEM Simulation}

The finite-element model of REMC-H was established in the electromagnetic-field finite-element software Ansoft Maxwell. Figures 7 and 8 show the simulated magnetic fluxes of REMC-H and REMC-R at different armature angles, respectively. For REMC-H, when the armature is in the middle position, the magnetic flux mainly passes through the radial air gap. When the armature rotates counterclockwise, most of the magnetic flux still passes through the radial air gap, while a small part of the magnetic flux passes through the additional axial air gap. The results of finite-element simulation are consistent with the working principle shown in Figure 3.

Some key structural parameters of radial and axial air gaps have a crucial influence on torque-angle characteristics. Proper selection of these parameters is helpful to improve torque-angle characteristics. In this study, four parameters, i.e., the height of the rectangular block of axial air gap $H_{r t}$, the radial air gap width $g$, the deviation angle of axial air gap $\alpha_{2}$, and the width of PM $B_{m s}$, were selected to carry out parametric design based on FEM simulation.

Here, $H_{r t}$ is defined as the distance from the top of the rectangular block of the axial air gap to the arc surface of the armature. Varying $H_{r t}$ is actually changing the effective area of the axial air gap. Figure 9 shows the effect of $H_{r t}$ on the torque-angle characteristics. The output torque increases, and the curve becomes flat gradually when $H_{r t}$ varies from $0.5 \mathrm{~mm}$ to $2.5 \mathrm{~mm}$, which indicates that $H_{r t}$ should be as large as possible.

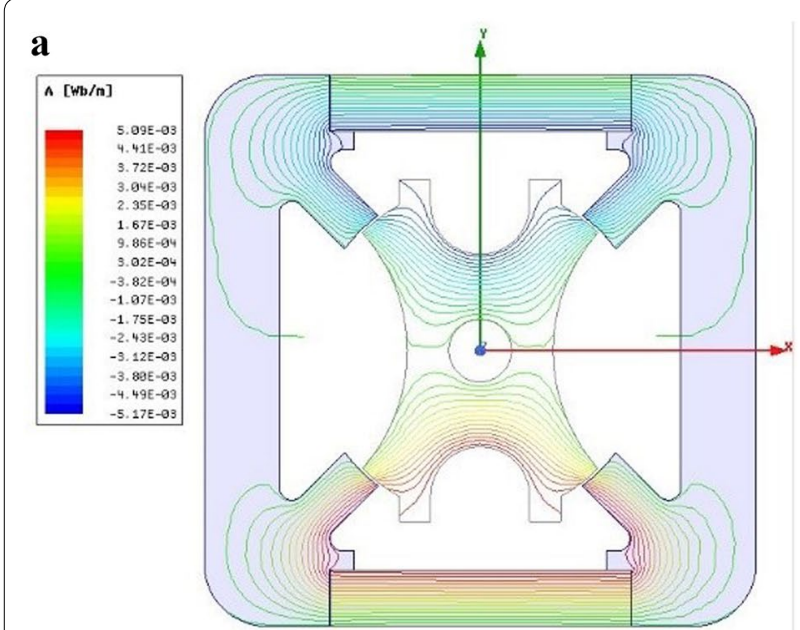

b

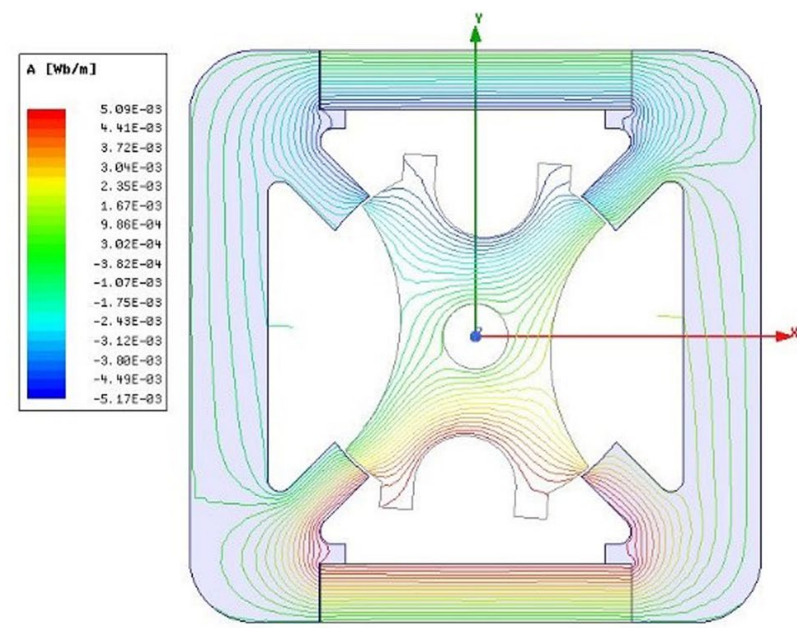

Figure 7 Magnetic flux of REMC-H

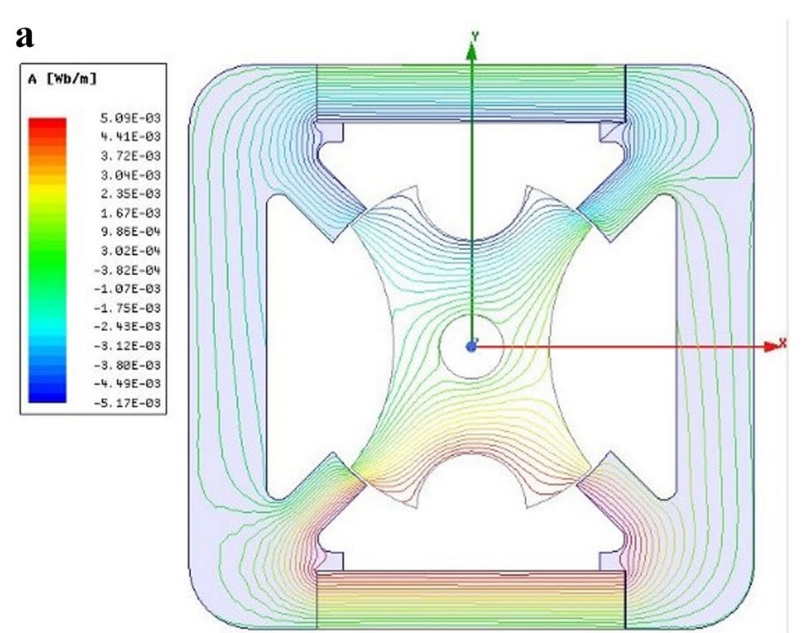

b

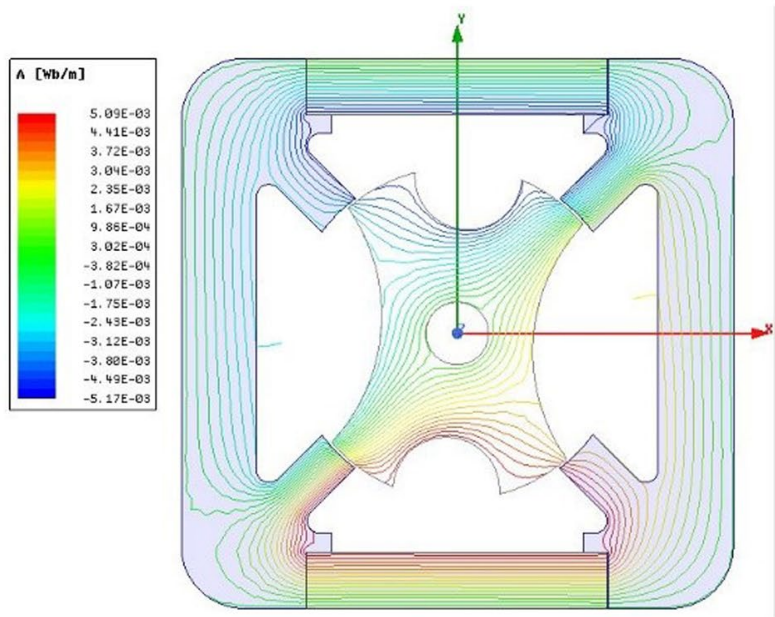

Figure 8 Magnetic flux of REMC-R 


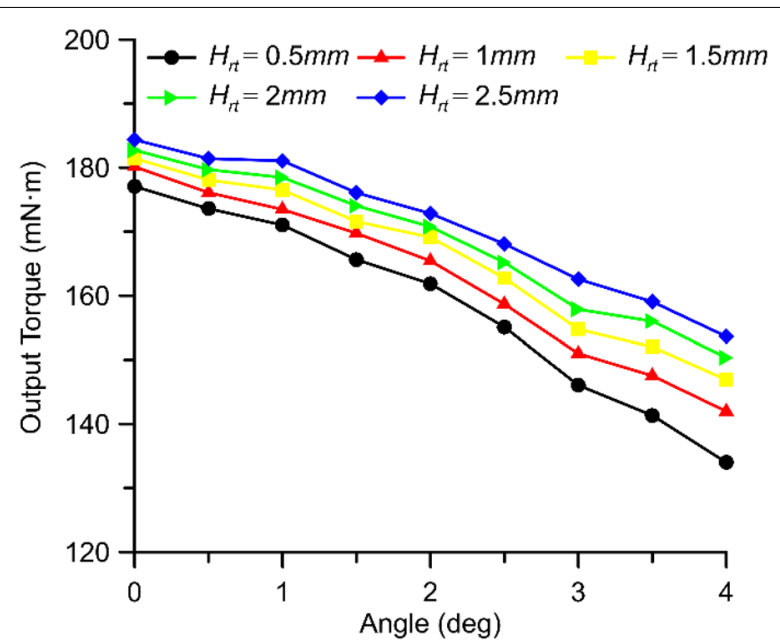

Figure 9 Effect of $H_{r t}$ on torque-angle characteristics

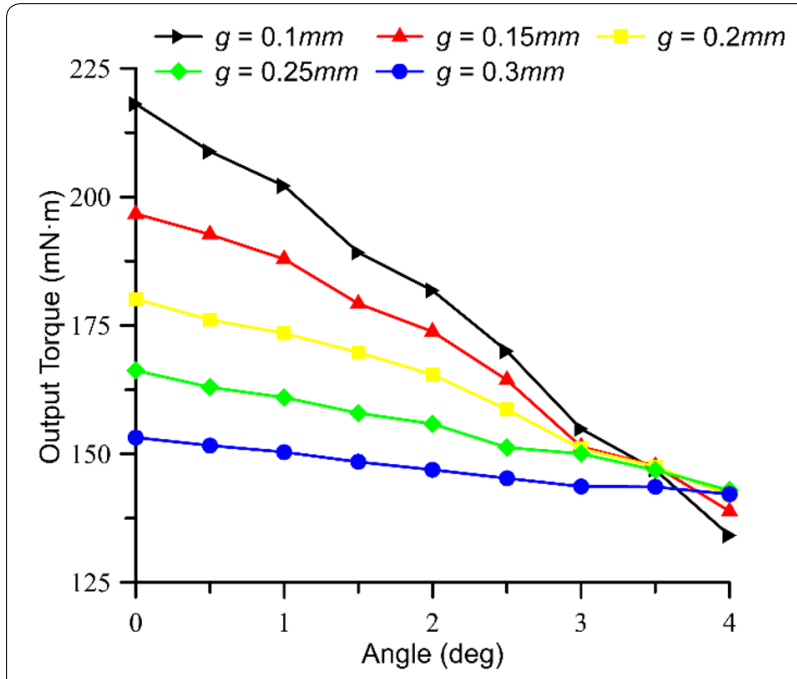

Figure 10 Effect of $g$ on torque-angle characteristics

In this work, $g$ is defined as the radius difference between the armature arc surface and pole shoe arc surface of the stator yoke, as shown in Figure 5. Figure 10 shows the effect of $g$ on the torque-angle characteristics. Reducing $g$ can greatly increase output torque, but, at the same time, the slope of the curve becomes steeper, which indicates that the choice of $g$ needs to be compromised together with consideration of manufacturing cost.

Here, $\alpha_{2}$ is defined as the angle between the rectangular block and vertical direction, as shown in Figure 6. Figure 11 shows the effect of $\alpha_{2}$ on the torque-angle characteristics. The influence of $\alpha_{2}$ on the initial torque is very limited, but, with the increase of armature angle,

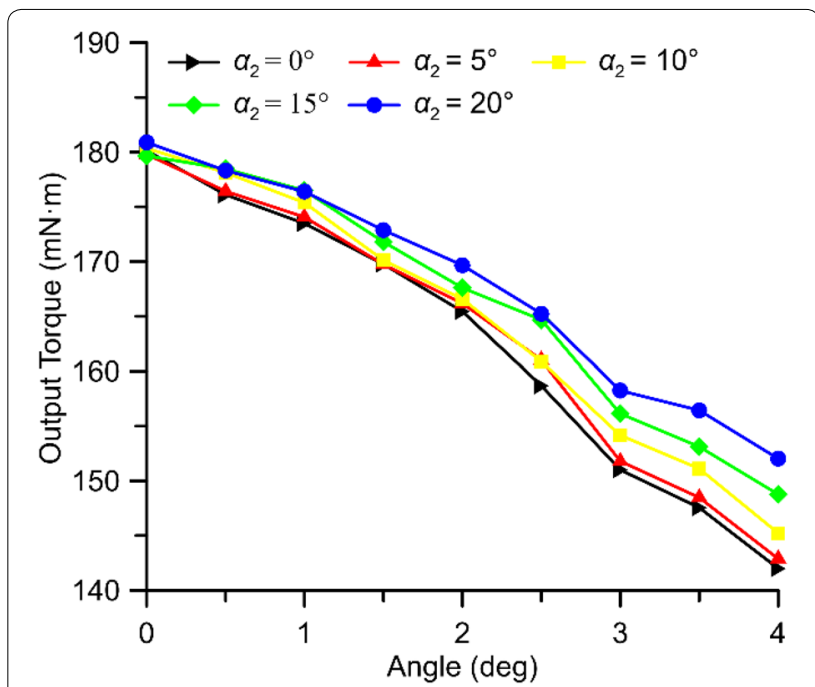

Figure 11 Effect of $a_{2}$ on torque-angle characteristics

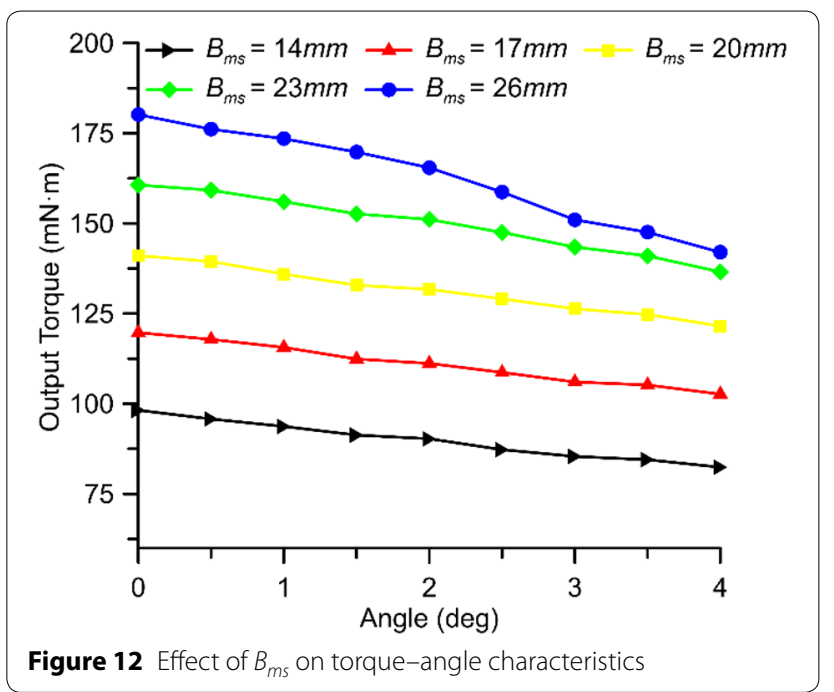

the torque-angle curve decreases slowly, which indicates that increasing $\alpha_{2}$ has a slight effect on curve flatness.

In the design of an electric-mechanical converter, it is crucial to match the control magnetic field and bias magnetic field. In some cases, it is unrealistic to increase the excitation current (such as preventing the coil from overheating), so the size of the PM must be adjusted to achieve optimal design. Here, $B_{m s}$ is defined as the width of the PM. Figure 12 shows the effect of $B_{m s}$ on the torque-angle characteristics. Generally, increasing $B_{m s}$ can improve the output torque. When $B_{m s}$ is less than $23 \mathrm{~mm}$, the curve is relatively flat. When $B_{m s}$ exceeds $26 \mathrm{~mm}$, the curve slope becomes steeper. Therefore, choosing $B_{m s}$ in the range of $20-23 \mathrm{~mm}$ can 
Table 1 Design parameters for prototypes

\begin{tabular}{ll}
\hline Parameter & Value \\
\hline Armature $($ REMC-R) length $L(\mathrm{~mm})$ & 26 \\
Armature $($ REMC-R) diameter $D(\mathrm{~mm})$ & 13.3 \\
Armature $(\mathrm{REMC}-\mathrm{H})$ length $L(\mathrm{~mm})$ & 26 \\
Armature $(\mathrm{REMC}-\mathrm{H})$ diameter $D(\mathrm{~mm})$ & 13.3 \\
Armature $\left(\right.$ REMC-H) height of rectangular block $H_{r t}(\mathrm{~mm})$ & 2 \\
Armature $(\mathrm{REMC}-\mathrm{H})$ width of rectangular block $B_{r t}(\mathrm{~mm})$ & 2.5 \\
Yoke height $H_{y r}(\mathrm{~mm})$ & 44 \\
Yoke length $L_{y r}(\mathrm{~mm})$ & 26 \\
Yoke width $B_{y r}(\mathrm{~mm})$ & 14 \\
PM length $L_{m s}(\mathrm{~mm})$ & 24 \\
PM width $B_{m s}(\mathrm{~mm})$ & 23 \\
PM height $H_{m s}(\mathrm{~mm})$ & 3 \\
Winding turns & 100 \\
\hline
\end{tabular}

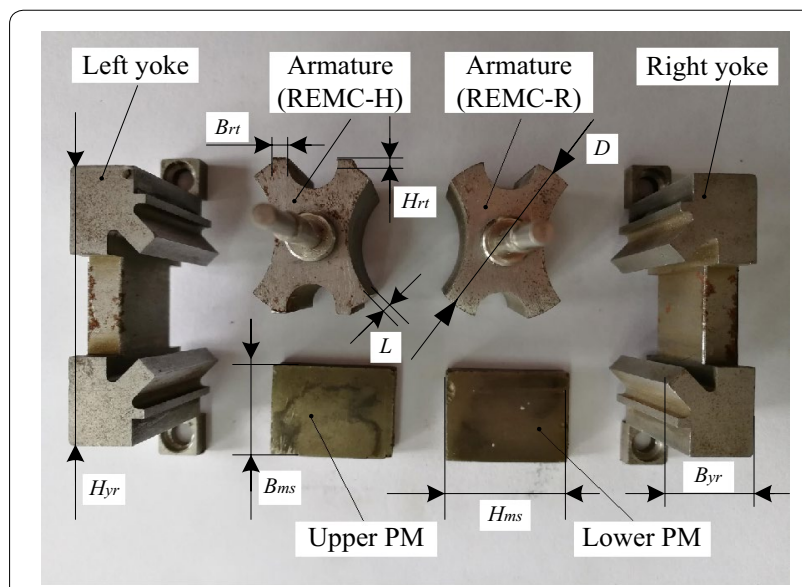

Figure 13 Main components of REMC-R and REMC-H

ensure both large output torque and the flatness of the torque-angle curve.

Based on the above-mentioned parametric design, the main structural parameters for prototypes were determined, as shown in Table 1 . The simulated torqueangle characteristics and step response also could be obtained by FEM simulation, whose results are discussed and compared with experiment results later.

\section{Experimental Study}

To validate the effect of the hybrid air gap on the regulation of torque-angle characteristics, experimental prototypes of REMC-R and REMC-H were both manufactured, as shown in Figure 13. Special test rigs for torque-angle characteristics and step response were built, as shown in Figures 14 and 15. For the torqueangle characteristic test, the stator yoke of prototype is mounted on plate $b$, while the armature components

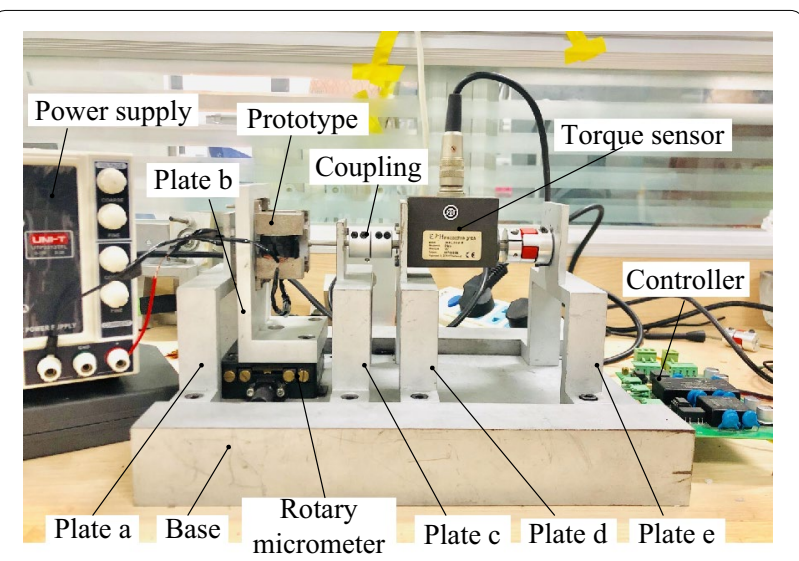

Figure 14 Test rig of torque-angle characteristics

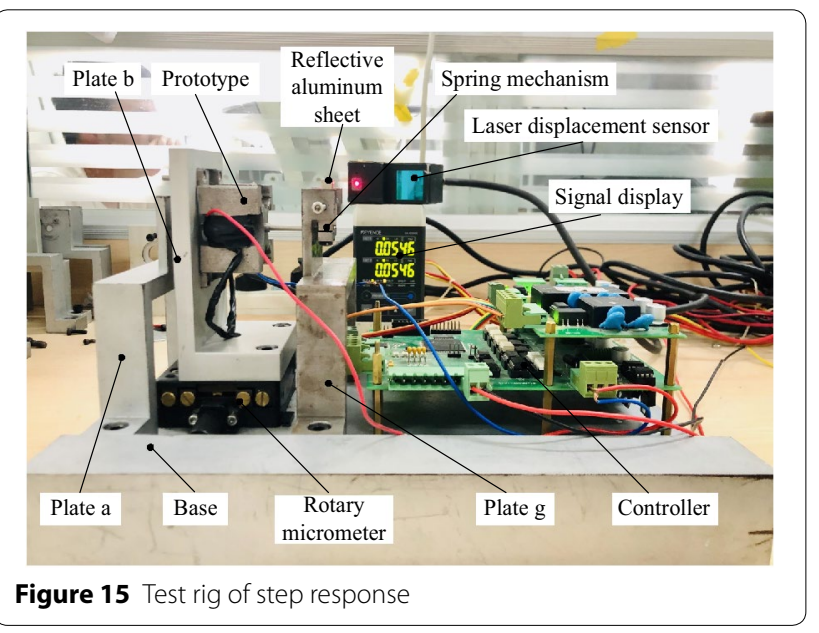

are mounted between plate a and plate $c$, where plate $\mathrm{b}$ is connected to a rotary micrometer with a measuring range from $-10^{\circ}$ to $10^{\circ}$ and accuracy of $0.1^{\circ}$. By manually adjusting the rotary micrometer, the stator can rotate relative to the armature. Also, a torque sensor is connected to the armature shaft through the coupling. Therefore, the rotary angle of the stator yoke and output torque can be read out by the micrometer and torque sensor, respectively. The torque-angle characteristics under different excitation currents and different armature angles were obtained.

For the step response test, the prototype installation was similar to that of the torque-angle characteristic test. To convert output torque into angular displacement, we designed plate $g$ with a spring balancing mechanism whose upper end was pasted to a reflective aluminum sheet. During testing, the input signal from the controller drove the prototype to rotate according to the control algorithm. The displacement of the aluminum sheet was measured by the laser displacement 


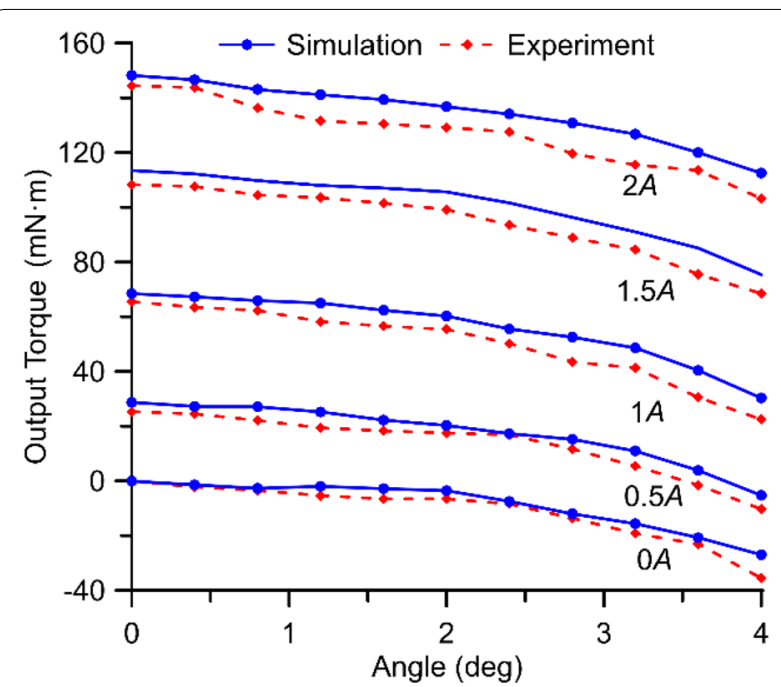

a REMC-R

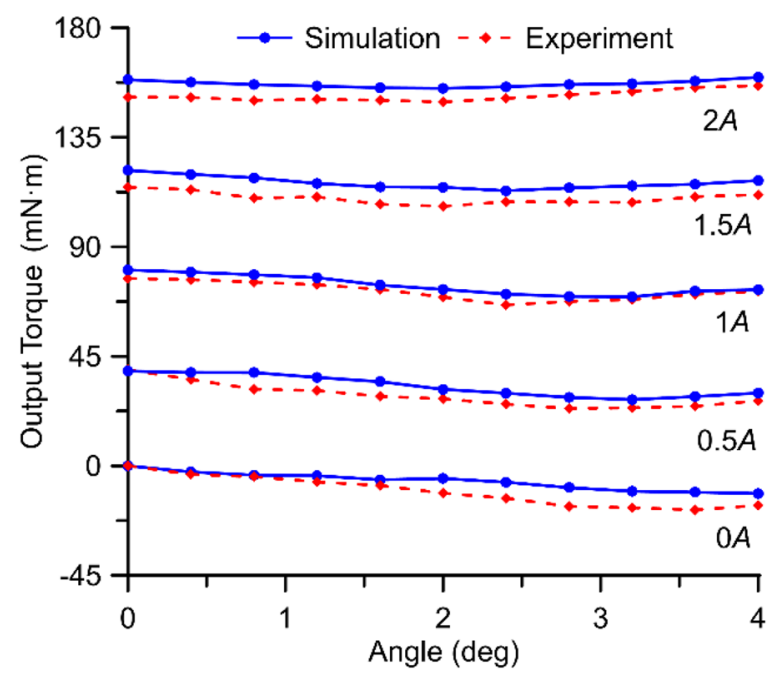

b REMC-H

Figure 16 Torque-angle characteristics of REMC-R and REMC-H

sensor, and the rotation angle of the prototype could be obtained indirectly.

Figure 16 shows a comparison between simulated and experimental results of the torque-angle characteristics of REMC-R and REMC-H under different excitation currents. Table 2 represents a detailed analysis of the experimental results, where $T_{\max }$ and $T_{\min }$ are maximum and minimum torque, respectively, $F$ is the torque fluctuation rate, and $G$ is the torque growth. $F$ and $G$ are defined as follows:

$$
F=\frac{T_{\max }-T_{\min }}{T_{\max }}
$$

Table 2 Analysis of experimental results of torque-angle characteristics

\begin{tabular}{llllll}
\hline $\boldsymbol{I}(\mathbf{A})$ & $\mathbf{0}$ & $\mathbf{0 . 5}$ & $\mathbf{1}$ & $\mathbf{1 . 5}$ & $\mathbf{2}$ \\
\hline $\begin{array}{l}T_{\max -R}(\mathrm{mN} \cdot \mathrm{m}) \\
(\mathrm{REMC}-\mathrm{R})\end{array}$ & 0 & 25.4 & 65.5 & 108.3 & 144.5 \\
$\begin{array}{l}T_{\min -R}(\mathrm{mN} \cdot \mathrm{m}) \\
(\mathrm{REMC}-\mathrm{R})\end{array}$ & -35.5 & -10.3 & 22.5 & 68.5 & 103.2 \\
$\begin{array}{l}T_{\max -H}(\mathrm{mN} \cdot \mathrm{m}) \\
(\mathrm{REMC}-\mathrm{H})\end{array}$ & 0 & 39.5 & 77 & 144.5 & 156.2 \\
$T_{\min -H}(\mathrm{mN} \cdot \mathrm{m})$ & -18.1 & 23.6 & 66.1 & 106.7 & 149.6 \\
$\begin{array}{l}\text { REMC-H) } \\
F_{R}(\%)\end{array}$ & - & 140.6 & 65.6 & 36.7 & 28.6 \\
$(\mathrm{REMC}-\mathrm{R})$ & - & 40.3 & 14.1 & 6.8 & 4.3 \\
$F_{H}(\%)$ & - & 55.7 & 17.6 & 5.7 & 8.1 \\
$\begin{array}{l}\text { REMC-H) } \\
G(\%)\end{array}$ & - & & &
\end{tabular}

$$
G=\frac{T_{\max -H}-T_{\max -R}}{T_{\max -R}}
$$

For REMC-R, the curve shows a monotonous downward trend with the increase of armature angle, which is consistent with the analysis of Eq. (11). When $I$ is $2 \mathrm{~A}$, $T_{\max -R}$ is approximately $144.5 \mathrm{mN} \cdot \mathrm{m}, T_{\min -R}$ is approximately $103.2 \mathrm{mN} \cdot \mathrm{m}$, and $F_{R}$ is $28.6 \%$. For REMC-H, the curve presents a nearly horizontal state with the increase of armature angle, which has good proportional control characteristics. Here, $T_{\max -H}$ is approximately 156.2 $\mathrm{mN} \cdot \mathrm{m}, T_{\min -H}$ is approximately $149.6 \mathrm{mN} \cdot \mathrm{m}$, and $F_{H}$ is $4.3 \%$. In addition, for both kinds of REMC, when $I$ increases, the torque fluctuation shows a downward trend. This indicates that, with the saturation of the magnetic circuit, the output torque is less and less affected by the rotation angle. Therefore, the REMC should be operated in the saturation state. In addition, with the same current, the output torque of REMC-H is always larger than that of REMC-R. With the increase of current, the torque growth rate decreases gradually. When $I$ is $2 \mathrm{~A}, G$ is approximately $8.1 \%$. Figure 17 shows the torque-current characteristics of both kinds of REMC, demonstrating their excellent linearity. The above analysis shows that REMC-H can obtain a flat torque-angle characteristic and has a larger output torque. The simulated and experimental results are in good agreement. The reason for the slight difference may be that the material characteristic parameters of the FEM software are different from the actual values, and there are some errors in machining.

Figure 18 is a comparison between simulated and experimental results of the step responses of REMC-R and REMC-H when the input current is $1.5 \mathrm{~A}$. Table 3 shows some parameters used to characterize the step response derived from Figure 18. The experimental rise 


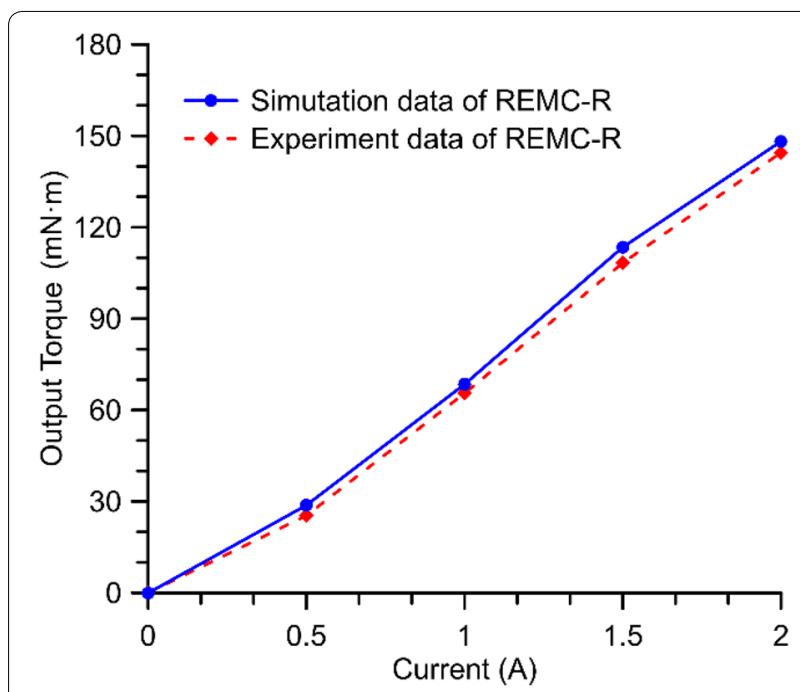

a REMC-R

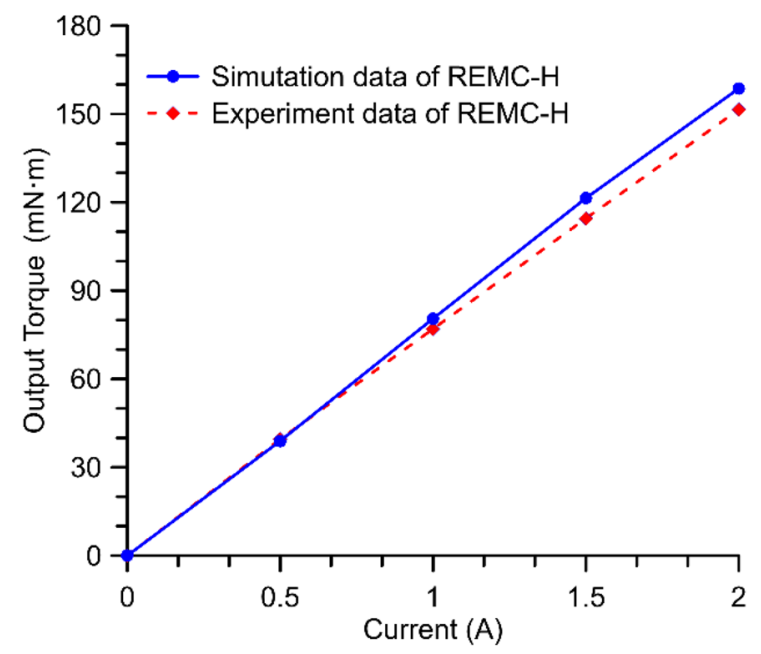

b REMC-H

Figure 17 Torque-current characteristics of REMC-R and REMC-H

time $T_{r}$ of REMC-H is $0.3 \mathrm{~ms}$ which lagged behind that of REMC-R, while the experimental armature displacement $\theta$ increases by approximately $0.3^{\circ}$ compared with REMC$\mathrm{R}$. This also proves that paralleling an additional axial air gap is beneficial to increasing the output torque from the point of view of dynamic response. Because the damping parameters and spring coefficients in the FEM simulation are constant, which is different from the actual situation, there are slight differences between the experimental and simulated curves, but they are acceptable.

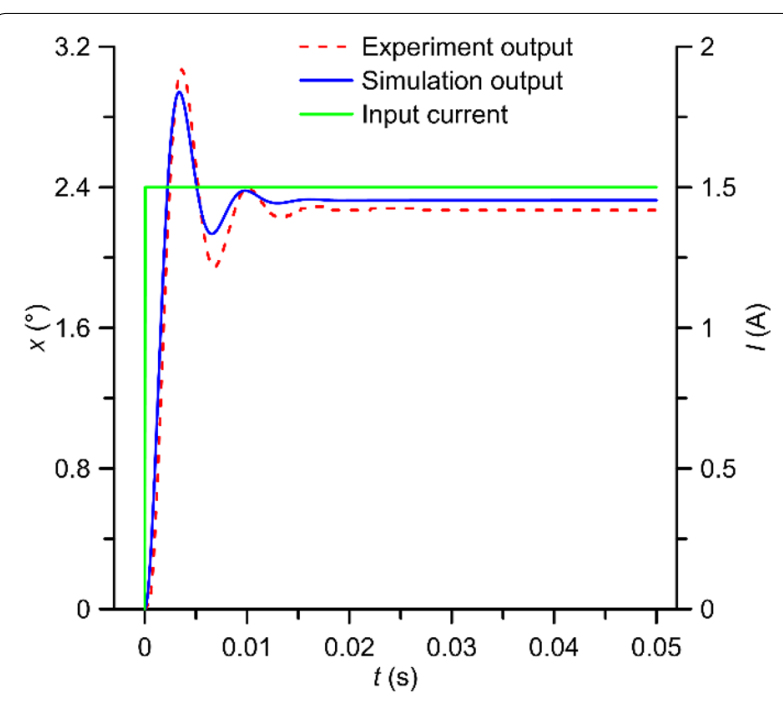

a REMC-R

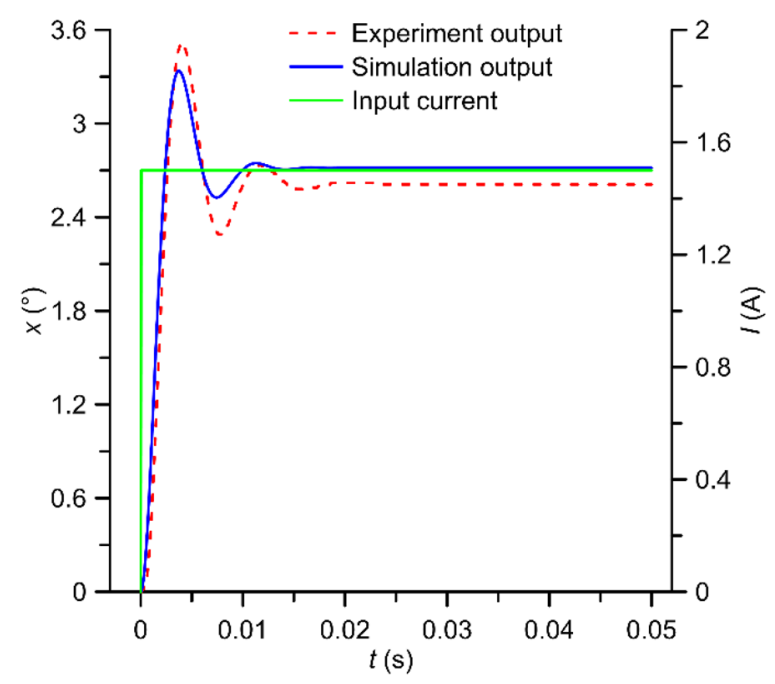

b REMC-H

Figure 18 Step response of REMC-R and REMC-H

Table 3 Experimental analysis of step response

\begin{tabular}{llll}
\hline & $\boldsymbol{T}_{\boldsymbol{r}}(\mathbf{m s})$ & $\boldsymbol{T}_{\boldsymbol{s}}(\mathbf{m s})$ & $\boldsymbol{\theta}\left(\mathbf{(}^{\circ}\right.$ \\
\hline REMC-R (simulated) & 3.3 & 12.6 & 2.3 \\
REMC-R (experimental) & 3.4 & 13.5 & 2.3 \\
REMC-H (simulated) & 3.7 & 13.5 & 2.7 \\
REMC-H (experimental) & 3.7 & 16.1 & 2.6 \\
\hline
\end{tabular}

\section{Conclusions}

(1) A simple and feasible regulation method for the torque-angle characteristics of an REMC based 
on a hybrid air gap was developed by paralleling an additional axial air gap to obtain flat torque-angle characteristics and increase output torque.

(2) The magnetic circuit analysis, FEM simulation, and experimental study of REMC-R and REMC-H prototypes were performed. The results show that the simulation and experimental results are basically in agreement with the analytical conclusions. The torque-angle characteristic of REMC-R is a monotonic descent curve, while the torque-angle characteristic curve of REMC-H is a flat curve, which validates the effectiveness of this method. In the torque-angle characteristic experiment for an excitation current of $2 \mathrm{~A}$, the maximum torques were 156.2 and $144.5 \mathrm{mN} \cdot \mathrm{m}$, respectively. In the step response experiment for an input current of $1.5 \mathrm{~A}$, the rise times were 3.4 and $3.7 \mathrm{~ms}$, respectively.

(3) The proposed regulation method provides a new approach for the design of proportional rotary electric-mechanical converters.

\section{Acknowledgements}

Not applicable.

\section{Authors' contributions}

BM was responsible for the main work of research in this article. $Y L$ deduced the analytical equations for REMC-R and REMC-H. XQ was in charge of some experiments. All authors read and approved the final manuscript.

\begin{abstract}
Authors' Information
Bin Meng, born in 1979, is currently an associate professor at Key Laboratory of Special Purpose Equipment and Advanced Processing Technology, Ministry of Education, Zhejiang University of Technology, China. He received his PhD degree from Zhejiang University of Technology, China, in 2013. His research interests include novel electro-mechanical converter and 2D hydraulic components.
\end{abstract}

Yongjiang Lai, born in 1993, is currently a master candidate at Key Laboratory of Special Purpose Equipment and Advanced Processing Technology, Ministry of Education, Zhejiang University of Technology, China.

Xinguo Qiu, born in 1978, is currently a senior engineer at Key Laboratory of Special Purpose Equipment and Advanced Processing Technology, Ministry of Education, Zhejiang University of Technology, China.

\section{Funding}

Supported by National Natural Science Foundation of China (Grant No. 51975524).

\section{Competing Interests}

The authors declare no competing financial interests.

Received: 5 September 2019 Revised: 7 January 2020 Accepted: 16 April 2020

Published online: 01 May 2020

\section{References}

[1] P Tamburrano, A R Plummer, E Distaso, et al. A review of electro-hydraulic servovalve research and development. International Journal of Fluid Power. 2019, 20(1): 53-98
[2] PTamburrano, A R Plummer, E Distaso. A review of direct drive proportional electrohydraulic spool valves: industrial state-of-the-art and research advancements. Journal of Dynamic Systems, Measurement, and Control, 2018, 141(2): 020801.

[3] R Amirante, E Distaso, P Tamburrano. Sliding spool design for reducing the actuation forces in direct operated proportional directional valves: experimental validation. Energy Conversion and Management, 2016, 119: 399-410.

[4] H Yan, Y K Ren, L Yao, et al. Analysis of the internal characteristics of a deflector jet servo valve. Chinese Journal of Mechanical Engineering, 2019, 32:31, https://doi.org/10.1186/s10033-019-0345-7.

[5] H Peng, J Z Wang, W Shen Wei, et al. Double fuzzy control with compensating factor for electronic-hydraulic servo valve-controlled system. Journal of Mechanical Engineering, 2017, 53(24): 184-192. (in Chinese)

[6] Y B Yin, J Y Yuan, JY Fu. Characteristics of two-stage relief valve with a series damping orifice in pilot valve front chamber. Journal of Jilin University, 2017, 47(1): 129-136. (in Chinese)

[7] C M Li, Y B Yin, M Y Wang, et al. Influence of high temperature on couples matching and characteristics of jet pipe electrohydraulic servovalve. Journal of Mechanical Engineering, 2018, 54(20): 251-261. (in Chinese)

[8] B Zhao, L Quan, J H Huang, et al. Characteristics of electro-hydraulic proportional valve with pilot pressure variation-displacement adjustment. Journal of Mechanical Engineering, 2017, 53(14): 195-201. (in Chinese)

[9] XW Peng, Y G He. Electro-hydraulic proportional servo control based on iterative learning algorithm. Journal of Mechanical Engineering, 2018, 54(20): 271-278. (in Chinese)

[10] XW Kong, X C Wang, X F Ruan, et al. Optimum design of fit clearance of proportional cartridge valve. Advances in Mechanical Engineering, 2018, 10(3): 1-9.

[11] J H Zhang, D Wang, B Xu, et al. Modeling and experimental validation of the time delay in a pilot operated proportional directional valve. IEEE Access, 2018, 6: 30355-30369.

[12] J Y Yuan, Y B Yin, L Lu, et al. Analysis of rotary direct drive electro-hydraulic pressure control servo valve. Journal of Mechanical Engineering, 2018, 54(16): 186-194. (in Chinese)

[13] J F He, X Chen, PY LU, et al. Theoretical analysis and experimental study on two-dimensional cartridge servo valve. Acta Aeronautica et Astronautica Sinica, 2019, 40(5): 422590. (in Chinese)

[14] Y Ren, J Ruan. Theoretical and experimental investigations of vibration waveforms excited by an electro-hydraulic type exciter for fatigue with a two-dimensional rotary valve. Mechatronics, 2016, 33:161-172.

[15] M Z Zhu, S D Zhao, P Dong, et al. Design and analysis of a novel doubleservo direct drive rotary valve with high frequency. Journal of Mechanical Science and Technology, 2018, 32 (9): 4313-4323.

[16] Y Liu, D Wang, D Zheng. Pulse wave generation method using rotary valve control. Journal of Mechanical Engineering, 2018, 54(20): 279-286. (in Chinese)

[17] Y Liu, G F Gong, HY Yang, et al. Regulating characteristics of new tamping device exciter controlled by rotary valve. IEEE/ASME Transactions on Mechatronics, 2016, 21(1): 497-505.

[18] Y Liu, S K Chen, D Zheng. Research status and prospect of high power wave generation technology. Journal of Mechanical Engineering, 2016, 52(24): 155-163. (in Chinese)

[19] Q Zuo, Q Q Lu, S Li, et al. Dynamic characteristics of 2d electro-hydraulic proportional directional valves with a ball-screw type axial compressiontorsion coupling. China Mechanical Engineering, 2017, 28(17): 2049-2055. (in Chinese)

[20] H B Xie, L Tan, J B Liu, et al. Numerical and experimental investigation on opening direction steady axial flow force compensation of converged flow cartridge proportional valve. Flow Measurement and Instrumentation, 2018, 62: 123-134.

[21] Y S Liu, Z Y Li. Hydraulic components and systems. 4th ed. Beijing: Mechanical Industry Press, 2019. (in Chinese)

[22] G M Wu, M X Qiu, Q F Wang, et al. New practical electro-hydraulic proportional technology. Hangzhou: Zhejiang University Press, 2006. (in Chinese)

[23] S H Wang, X L Xiao, G Y Xiong. Direct current electromagnets with constant traction characteristic. Journal of Mechanical Engineering, 2008, 44(2): 244-247. (in Chinese) 
[24] A Ferrari, P Pizzo, M Rundo. Modelling and experimental studies on a proportional valve using an innovative dynamic flow-rate measurement in fluid power systems. Proceedings of the Institution of Mechanical Engineers, Part C: Journal of Mechanical Engineering Science, 2018, 232(13): 2404-2418.

[25] S Wu, Z X Jiao, L Yan, et al. Development of a direct-drive servo valve with high-frequency voice coil motor and advanced digital controller. IEEE/ ASME Transactions on Mechatronics, 2014, 19: 932-942.

[26] J H Buscher, E Amherst. Complier: US, 5679989. 1997-10-21 [2019-07-16]. http://www.pat2pdf.org/patents/pat5679989.pdf.

[27] H S Jeong. Novel torque motor model for performance design and analysis of a servo valve with permanent magnets. Journal of Mechanical Science and Technology, 2018, 32(5): 2253-2259.
[28] J I Montagu. Complier: US, 3959673. 1976-05-25[2019-07-16]. http:// www.pat2pdf.org/patents/pat3959673.pdf.

[29] I Takaharu, K Hisasi, H Kyo, et al. Complier: US, 4496134. 1976-05-25[201907-16]. http://www.pat2pdf.org/patents/pat4496134.pdf.

[30] J Cui. Research on key technology of rotary direct acting electro-hydraulic servo valve. China: Zhejiang University, 2008. (in Chinese)

[31] G Q Zhang, JY Lin. A new type of electromechanical converter-high voltage bipolar proportional electromagnet. Hydraulic and Pneumatic, 1986, 3: 15-18. (in Chinese)

[32] J Cui J, F Ding, Q P Li. Novel bidirectional rotary proportional actuator for electrohydraulic rotary valves. IEEE Transactions on Magnetics, 2007, 43(7): 3254-3258.

\section{Submit your manuscript to a SpringerOpen ${ }^{\circ}$ journal and benefit from:}

- Convenient online submission

- Rigorous peer review

- Open access: articles freely available online

- High visibility within the field

- Retaining the copyright to your article

Submit your next manuscript at $\boldsymbol{\nabla}$ springeropen.com 